Questions vives

\section{Questions Vives}

Recherches en éducation

$\mathbf{N}^{\circ} 34 \mid 2020$

Expériences vécues et surgissement d'événements : une écriture du sensible en recherche biographique

\title{
Quelle posture pour le chercheur en situation d'entretien dans le milieu carcéral avec des personnes dites « en grande vulnérabilité »?
}

What is the position of the researcher in a prison interview with people who are

"highly vulnerable"?

\section{Marie Hèlène Verneris}

\section{Q OpenEdition}

\section{Journals}

\section{Édition électronique}

URL : https://journals.openedition.org/questionsvives/5248

DOI : 10.4000/questionsvives. 5248

ISSN : 1775-433X

Éditeur

Université Aix-Marseille (AMU)

\section{Édition imprimée}

Date de publication : 18 décembre 2020

ISBN : 978-2-912643-58-2

ISSN : $1635-4079$

\section{Référence électronique}

Marie Hèlène Verneris, «Quelle posture pour le chercheur en situation d'entretien dans le milieu carcéral avec des personnes dites « en grande vulnérabilité »? », Questions Vives [En ligne], №34 I 2020, mis en ligne le 18 décembre 2020, consulté le 11 octobre 2021. URL : http://

journals.openedition.org/questionsvives/5248; DOI : https://doi.org/10.4000/questionsvives.5248

Ce document a été généré automatiquement le 11 octobre 2021.

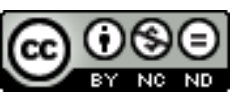

Questions Vives est mis à disposition selon les termes de la licence Creative Commons Attribution -

Pas d'Utilisation Commerciale - Pas de Modification 4.0 International. 


\section{Quelle posture pour le chercheur en situation d'entretien dans le milieu carcéral avec des personnes dites « en grande vulnérabilité »?}

What is the position of the researcher in a prison interview with people who are "highly vulnerable"?

Marie Hèlène Verneris

\section{Quelle posture pour le chercheur en situation d'entretien dans le milieu carcéral avec des personnes dites « en grande vulnérabilité » ?}

1 Le récit considéré comme une production scientifique est pour le chercheur la forme la plus accessible et communicable à autrui. Divers protocoles méthodologiques, aux degrés variables de technicité et de sophistication existent pour permettre au chercheur, poursuivant sa quête humaine de compréhension et de connaissance des autres dans leur singularité, de raconter ce que le récit des autres lui apprend. Si les chercheurs en sciences humaines, sociologues, ethnologues sont nombreux à s'interroger sur leurs pratiques d'enquêtes qualitatives, peu de travaux s'intéressent au recueil de données sur un terrain sensible. La recherche biographique sur l'usage de laquelle porte notre réflexion ne peut se satisfaire de bricolages méthodologiques s'appuyant sur la combinaison de méthodes et d'approches plus ou moins formelles, surtout si celle-ci intervient auprès de personnes se trouvant en grande vulnérabilité.

Dans ce cadre spécifique, qui fait du récit le cœur de sa réflexion théorique, nous ne pouvons que constater l'importance de la dimension éthique. Le code de déontologie de bonne pratique en recherche qualitative selon Janner-Raimondi, (à paraître 2021) suppose, pour le recueil du récit, une démarche compréhensive qui induit la pratique 
d'une écoute attentive et active, d'une attitude d'empathie envers l'autre et d'une prudence à ne pas blesser l'autre. Mais qu'en est-il de la recherche et du chercheur dès lors que celui-ci se trouve en milieu dit sensible? Le terrain du sensible inviterait-il le chercheur à reconsidérer la question de sa posture face à autrui ? Comment apprend-il de cette expérience et, comment s'est-il construit son expertise? Ces questions s'appuient sur une démarche de recherche qualitative qualifiée de recherche heuristique et appelle une réflexion épistémologique innovante. Nous ne prétendons pas apporter ici des recettes toutes faites, mais seulement de proposer des éléments de réflexion à partir de notre angle de vue singulier sur la base des expériences d'entretiens réalisés dans un des mondes sociaux les plus disqualifiés, l'univers carcéral.

3 Si la difficulté apparaît inhérente à l'objet étudié ou induite par le contexte de la recherche, notre analyse engagée ci-après s'orienterait vers la capacité du chercheur à faire évoluer ses pratiques selon les terrains inédits voire risqués au sein desquels il s'inscrit. Comme le conseille Boumaza (2007) l'intérêt serait d'identifier les conséquences sur les investigations en terrains difficiles, et sur les modifications des habitus du chercheur exposé. Nous qualifions de terrains sensibles des lieux et zones où sont convoquées violence et insécurité, au sein desquels vivent des groupes sociaux ou des personnes marginalisées et/ou stigmatisés qui ne se laissent pas facilement approcher du fait de leurs activités ou de leurs manières de vivre considérées comme socialement illégitimes.

4 Pour avoir toutes vécues dans l'univers carcéral, au sein d'un environnement réputé marginal et dangereux, un lieu d'exclusion par excellence où coexistent toutes formes de précarités et vulnérabilités, les personnes, dont il est question dans la recherche que nous avons réalisée, ont toutes connu des situations extrêmes, qui font écho à des épreuves dont elles ne se sont pas ou difficilement remises. Les questions légitimes que le chercheur se pose se trouvant sur le terrain du sensible sont : comment appréhender l'expérience de "situations limites de l'existence", dont la personne ne revient pas indemne et comment s'immerger dans un milieu dit sensible où les souffrances morale, physique, psychologique et sociale, s'inscrivent au quotidien. Il est en effet des situations vécues qui frôlent les limites de ce qui peut se dire et ébranlent à ce point les personnes qui les vivent, qu'elles en viennent à ne plus savoir quel sens donner à ce qu'elles ont vécu, et quels sens donner à leur vie d'après. Evoquer l'expérience sensible via le langage conduit la personne à s'ouvrir à l'autre au risque de se retrouver face à face avec des moments et des évènements passés douloureux qui ont fait trace, dans sa mémoire et dans sa chair, laissant des marques indélébiles pouvant faire émerger d'intenses charges émotionnelles. Les enjeux d'une prise en compte de l'expérience sensible sont d'autant plus importants que ces personnes ont souvent été stigmatisées et leur parole discréditée. Il est, par ailleurs, légitime pour le chercheur de s'interroger sur la forme scripturale scientifique la mieux adaptée à restituer les récits exprimés, explorant les caractéristiques d'une écriture du sensible spécifique à la recherche qualitative de type compréhensive.

5 Une grande majorité de chercheurs en sciences humaines et sociales fondent leur posture relationnelle durant l'entretien sur la notion de non-directivité qui nous vient du psychologue nord-américain Carl Rogers (2015). Un concept issu du courant de la psychologie humaniste que son inventeur lui-même sera le premier à critiquer (Rogers, 1972) en dénonçant ses limites au regard des difficultés à l'appliquer. L'attitude de non 
directivitê du chercheur a été souvent confondue avec un laisser-faire ou avec une attitude de passivité qui serait susceptible d'être interprétée par la personne qui raconte son histoire comme un manque d'intérêt, voire une marque de rejet. Rogers (2001) la remplacera par ce qu'il appellera l'approche centrée sur la personne, soit une manière d'être qui amène la personne à découvrir ses propres potentialités, à devenir directif de son existence, d'être sa manière d'être. Un fondement qui exige l'installation de trois conditions interpersonnelles nécessaires et préalables entre le chercheur et la personne. La première sera celle de l'empathie, soit la capacité à comprendre le monde de l'autre, d'entrer dans la subjectivité du monde de l'autre pour voir et surtout comprendre comment l'autre vit sa relation au monde. La seconde sera la congruence, soit la conscience et la transparence du chercheur et la troisième sera la considération positive et inconditionnelle qui suppose pour le chercheur une acceptation de l'autre sans jugement ni évaluation. Le propre de l'approche rogérienne n'est pas d'agir sur le problème, moins encore de chercher une solution, mais de se focaliser sur la personne en cherchant à créer le climat favorable qui facilitera son ouverture et son épanouissement. Nous sommes bien ici dans la recherche d'une manière d'être, soit d'une posture d'accompagnement qui va amener l'autre à être vraiment lui-même. Au regard de notre expérience de terrain, souvent confrontée au recueil de récits traumatiques, cette approche centrée sur la personne mérite d'être développée et approfondie lorsqu'elle se situe sur le terrain du sensible.

Au-delà de la dimension d'écoute de la parole de l'autre, l'écoute de ses hésitations comme de ses silences et de l'empathie dont le chercheur fait preuve, sa posture dans le cadre de la recherche biographique en terrain sensible s'organise autour de l'attention portée au fondement de l'expérience vécue de la personne, de lui accorder de la valeur, à travers sa manière d'être au monde, son rapport aux autres, la relation à soi et à l'historicité de sa vie. Abordant cette question, Ricœur (1991) conçoit la primauté de l'éthique sur la morale, en évoquant le souci de soi et le souci de l'autre pour qualifier la visée éthique; la morale recouvrant plutôt le côté obligatoire, les obligations et normes. Loin de se conformer stricto sensu à une méthodologie plutôt qu'à une autre, c'est comme le prévoit Janner-Raimondi (à paraître 2021) à travers une éthique de la délicatesse cherchant à dire ce qui s'est produit sans dire, tout en disant, pour éviter de froisser, voire de nuire à la personne ${ }^{1}$ que nous développons une attitude favorisant le dialogue et la confiance afin que le sens soit partagé. Ainsi pour accueillir la parole et appréhender le mieux possible la dimension du sensible, nous nous sommes inscrit « en contrebande " des règles fondamentales de l'entretien de biographisation, laissant une large place à la créativité qui émerge en temps réel de l'expérience même. Nous aménageons intuitivement un espace de rencontre qualitatif adapté à la personne selon sa situation sociale et judiciaire en adoptant une posture à la fois perceptive et ouverte au champ des possibles ; une posture qui s'est affinée et éprouvée au fil des entretiens.

Notre pratique de l'écoute et l'accueil du sensible est aussi passée par un apprentissage des gestes, un apprentissage du sensoriel dans la maîtrise progressive du ressenti chez la personne comme en nous. Accueillir le sensible appelle une double posture de recherche pour laquelle l'implication du chercheur dans la relation avec l'autre est incontournable, toutefois celle-ci ne peut s'engager que dans une dimension imprégnée de neutralité, une dualité fondamentale.

8 Peu de chercheurs avertis parlent de la sincérité et l'authenticité du chercheur, pour autant c'est ce que nous nous attachons à démontrer pour installer la personne dans 
une posture propice à raconter sa vie. Nous insistons sur le caractère indépendant de notre recherche, nous ne sommes ni subventionné, ni rémunéré, ni affilié à un organe du Ministère de l'Intérieur, de la Justice ou de l'administration pénitentiaire. Nous présentons notre projet sous son aspect universitaire destiné à mieux comprendre le sens que les personnes donnent à leurs vécus, dès lors qu'elles ont traversé au moins une expérience en prison, avec l'objectif d'étudier l'impact du parcours carcéral sur leur existence. Cette manière de nous détacher de l'institution nous positionne sans la moindre ambiguïté en dehors de l'univers dont ils se méfient et a rencontré un écho favorable auprès des dix personnes concernées du fait de l'intérêt que nous portions à leurs parcours. Les réactions obtenues étaient: "pour une fois qu'on s'intéresse à nous » ou « ça fait du bien d'être entendu, pour vous au moins on existe ».

9 Nous avons réalisé dix entretiens de biographisation, "dans les murs » et " hors les murs », organisant des espaces de rencontre humaine et individuelle qui donnent à la personne l'occasion de se raconter et de se comprendre. Passer du temps avec les personnes dont nous avons essayé de saisir qui elles sont, comment elles se représentent, comment elles ont vécu leurs expériences biographiques carcérales, et de quelle manière elles trouvent leur place dans un contexte social, historique et culturel, nous a permis de nous familiariser avec le monde de la prison et des personnes qui y vivent, en établissant des relations de collaboration et de sincérité. D'une certaine façon, cette posture impliquant un processus de formation à savoir un processus de subjectivation, ouvrant le champ des possibles nous a grandi et enrichi.

Le choix s'est réorienté vers l'explication « d'une consigne de départ permettant de déclencher la production discursive de la personne en formulant quelques pistes d'interrogations indicatives", (Demazière, 2008, p.15). Il s'agissait également d'interrompre le moins possible la parole donnée pour la laisser «se libérer ». Pour commencer, nous invitons la personne à démarrer son récit de vie en lui demandant de nous parler d'elle, de qui elle était avant l'incarcération (la première s'il y en a eu plusieurs), de nous raconter comment elle a vécu la prison, et de terminer par ce qu'elle pense d'elle aujourd'hui si elle est sortie, ou ce qu'elle envisage et espère dans le cadre de sa sortie si elle est toujours incarcérée. Le récit de vie s'inscrit donc autour de trois temps différents, avant, pendant et après l'enfermement (pour celles qui sont en liberté). Nous posons peu de questions, uniquement dans les cas où le discours est désynchronisé, pour veiller à respecter la chronologie des évènements, et seulement si la personne s'égare et aborde d'autres thèmes hors sujet. Éviter une succession de questions préétablies nous permet d'espérer recueillir de la personne une parole spontanée exclusivement centrée sur elle-même. Si quelques réajustements sont parfois nécessaires, nous "laissons venir la parole" pour que celle-ci soit la plus ouverte possible, que la personne s'exprime avec ses mots à elle et dans l'ordre qui lui convient. Dans notre relation au sensible, le « laisser venir à soi » s'entend comme « un savoir attendre » exigeant de ne rien préjuger, rien anticiper et de ne rien suggérer à l'autre pour ne pas faire intervenir notre volonté qui pourrait aller à l'encontre de la parole spontanée (Bois \& Austry, 2007, p. 10). Les caractéristiques spécifiques d'une écriture du sensible nous imposent de fixer ultérieurement un autre temps, consacré à la clarification de certains passages dont la compréhension nous a échappé. Cette étape nous permet de signifier à la personne que l'entretien est aussi l'occasion d'une coopération-collaboration, d'un travail concerté pour atténuer le sentiment d'asymétrie. Un dernier moment enfin est consacré à la relecture de la totalité de 
l'entretien retranscrit pour s'assurer de la conformité du discours et proposer une mise en forme du témoignage la plus fidèle possible.

11 Le terrain du sensible conduit le chercheur à anticiper une réflexion nourrie sur la manière dont il voit et considère le monde. Il l'invite à se réinterroger sur son être, sur le soi, sa vie, et le monde, l'éclairant sur la façon dont il vit et perçoit son rapport à soi et aux autres. Nous soutenons l'idée selon laquelle les auteurs de délits et crimes ne sont pas des êtres à part, dénués de certaines capacités intellectuelles, présentant des troubles de la personnalité et incapables de choix ${ }^{2}$, mais des personnes proches de nous, ordinaires, qui nous ressemblent, leurs actes résultant de choix rationnels. Par ailleurs nous considérons que leurs actes délictueux ayant déjà été jugés, la justice étant passée, nous nous abstenons de porter le moindre jugement de valeur sur ce qui nous est raconté quel qu'en soit le récit. Caractérisée par le souci du respect de l'autre, cette prégnance culturelle d'une éthique du non-jugement s'est développée au fil de notre expérience de formateur-accompagnant en prison.

Nous savons que parler de soi n'est pas chose facile, bien plus encore lorsqu'il s'agit d'expériences socialement stigmatisées ou disqualifiées et dès lors que nous touchons à une dimension qui appartient au privé et à l'intime. Le terrain exploratoire dans lequel nous évoluons nous impose d'écouter et de partager des situations qui relèvent parfois de l'extrême. L'éthique de notre démarche nous rappelle que la quête de la vérité n'est pas l'objet même de notre recherche. Nous partageons donc l'idée que les personnes conservent une part d'intimité, d'ambivalence et d'obscurité qui demeurent inaccessible à la recherche. Il nous faut tenir compte également du fait que, pour certaines personnes, construire un discours autour d'actes déviants commis, donner sens à des processus dans lesquels elles sont peut-être encore engagées au moment de l'entretien, sont des situations complexes qui peuvent susciter des justifications, voire des omissions. Nous recueillons la parole telle qu'elle nous est livrée par son auteur, l'une des difficultés de cette recherche étant notre capacité en tant que chercheur à recevoir sans objecter, à écouter sans prendre position et à analyser sans prendre parti. D'expérience, nous savons que la démarche de biographisation, permettra par le processus de subjectivation qui la caractérise, une meilleure compréhension des conduites des individus à la lumière des définitions qu'ils en donnent, que leur analyse de la réalité soit juste ou non. Comme le formule Laub, nous ne sommes pas «à la recherche de la vérité objective mais de leur vérité » $(2015$, p. 146) et comme le précise Delory-Momberger (2014), c'est en biographiant leur vie que les individus pourront espérer comprendre les interactions et les évènements qui les ont marqués et prédisposés à telle ou telle transgression.

Le terrain du sensible renvoie également le chercheur à penser la place et le rôle qu'il occupe dans les expériences vécues retransmises dans un corps à corps touchant et touché ; à considérer notre sens moral et notre crédibilité, à questionner notre responsabilité, notre objectivité et enfin à évaluer notre capacité à voir la souffrance sans pouvoir la réduire, sans prendre la défense et sans prendre parti pour les plus faibles ou pour l'institution qui les enferme. Nous avons constaté qu'en adoptant une attitude trop favorable aux détenus, qui aimeraient obtenir notre adhésion face à l'institution serait inappropriée. Aux conflits moraux que nous ressentons face aux situations génératrices de malaises, ou de troubles, s'ajoutent une relation dissymétrique qui empêche la réciprocité entre égaux et circonscrit l'accès à la réalité. Si nous partageons avec Rostaing (2010, p. 23) l'idée que «l'on ne sort jamais indemne 
d'une recherche longue en prison " ce n'est pas d'avoir craint pour notre sécurité personnelle, ni pour avoir souffert des conditions et contraintes sécuritaires permanentes, ni pour la gêne d'être une femme parmi un groupe de quinze détenus tous enfermés à double tour sans surveillance dans une salle de classe pendant plusieurs heures, mais plutôt pour y avoir vu et avoir été témoin de faits de violence inexplicables sur des personnes en état de grande vulnérabilité dont la situation de faiblesse ne justifiait pas l'intensité de la violence. Nous sommes confrontée régulièrement à un univers aux pratiques perverses et violentes dont les conduites, sur une population disqualifiée et stigmatisée soulèvent à bien des moments notre indignation. C'est avec une grande pudeur que nous avons évoqué dans notre thèse ${ }^{3}$ les situations critiques auxquelles nous avons dû faire face, parce que nous nous sommes trouvée trop souvent seule, impliquée face à la souffrance d'autrui avec le sentiment d'être prise en otage entre l'envie de résister à la déshumanisation produite par le système de l'institution carcérale, et l'obligation du respect des règlements garantissant la ligne de conduite institutionnelle attendue. Repenser à ses nombreuses situations de terrain (assister à une baston entre détenu et surveillants) qui font écho aux malaises ressentis sur le terrain renforce l'intérêt du chercheur à réfléchir sur la gestion et l'intégration de ses émotions dans la recherche.

\section{Qu'en est-il de notre responsabilité ?}

14 Notre responsabilité est engagée dans le sens où nous sommes le « libre témoin » ou le «moins libre complice» ayant pu accéder à un univers dont l'opacité est symboliquement représentée par l'épaisseur de ses murs d'enceinte. Nous avons entendu, vu et recueilli les paroles, les histoires de ceux qui rarement se sont exprimés, parce que relégués à leurs méfaits, leur parole est refusée. Comme Garfinkel (1967) nous nous demandons s'il nous faut «abdiquer de cette responsabilité d'avoir vu des choses peu remarquées, et peut être compris des choses peu élaborées?». Nous nous sommes également interrogée sur notre capacité à rester objective dans une réalité aussi dure face à ce que nous voyons et entendons autour de nous. A l'écoute des récits de Claude (qui a passé 35 années de sa vie en prison « toujours debout »), Liana (accusée à tort, condamnée à 8 ans de prison à l'étranger, "battue violée par une gardienne ») ou Jordan (qui a donné 60 coups de couteaux à son rival et se dit "prêt à recommencer"), une fois les effets de sidération passés, effets dus à l'horreur d'une situation racontée associée au vécu de la souffrance, nous savons que les mots nous ont parfois manqués. Nous nous sommes questionnée de façon récurrente sur la «bonne façon » de recevoir la souffrance de l'autre. Comment exprimer à la fois la compassion face à la peine et la gêne ressentie lorsque l'intimité est mise à nue ? Nous avons encouragé la parole, pour laisser les mots venir à nous, nous avons invité la personne à prendre son temps pour dire, tout en confirmant que nous avons bien entendu ce qu'elle venait de dire, consciente du lien infiniment sensible qui nous relie que nous ne pouvons laisser s'échapper, et qui doit être maintenu entre nous pour que cette offre de parole ne verse pas dans le voyeurisme, et pour qu'elle ne soit pas perçue comme un intérêt morbide de notre part. Il nous a fallu accepter de ne rien avoir à dire, à commenter ou à répondre des évènements qui nous sont racontés avec tant de détails, de précautions et d'efforts de mise en scène. Nos silences parfois lourds et pesants, marquent à la fois la retenue et la discrétion dont nous faisons preuve, le respect qui s'impose face à la douleur de l'autre, mais aussi notre incapacité à éprouver ce que l'autre ressent parce que nous ne 
pouvons pas nous mettre à sa place. Souvent nous nous sommes demandée si toutes ces émotions plus ou moins bien contrôlées impacteraient notre recherche et ses résultats, et dans quelle mesure celle-ci pourrait en être affectée (Verneris, 2018). Pour autant, aucune connotation d'angélisme n'a jamais détourné notre jugement, de même que minimiser ou légitimer certains actes commis décrits dans les récits aurait été inapproprié ; «les préceptes moraux et les principes qui nous habitent, sont autant d'armes pour faire la part des choses entre le bien et le mal, et entre le permis et l'interdit » (p. 20).

\section{Qu'en est-il de l'éthique de notre démarche?}

15 L'éthique de notre démarche de biographisation pose la question de la décence/ indécence envers les personnes dont la vulnérabilité et la fragilité sont très largement identifiées. Nous pensons plus précisément à cette distance de fait qu'impose notre situation de chercheur. Le malaise, la gêne, ou encore la peur dit Rostaing (2010, p. 23), "de ne pas être à sa place ». L'indécence pour la différence ou l'écart entre le monde que nous habitons et où nous vivons et celui de la personne détenue, ce croisement entre deux mondes qui se regardent et s'observent, aimeraient bien se comprendre mais ne s'appartiennent pas et jamais ne se confondent. Nous ne mesurons jamais assez les risques d'accentuer la souffrance de l'autre, de faire rejaillir à vif chez l'autre ce qu'il aurait mieux fait d'oublier, nous n'évaluons jamais assez les réels dangers de s'exposer au lieu de se protéger. Pour toutes ses raisons, l'entretien avec des personnes particulièrement fragilisées, vulnérables ou affaiblies est, d'un premier abord indécent. Le sentiment d'impuissance, celui de l'indignation ou de la compassion sont régulièrement invités. Celui de l'injustice également nous questionne : et si ces hommes étaient issus de milieux favorisés, s'ils avaient eu accès à une meilleure éducation, auraient-ils commis ce pourquoi ils sont en prison aujourd'hui? Les tensions liées aux conflits moraux et aux dilemmes que nous ressentons, éprouvent les missions et la posture du chercheur sur le terrain.

16 Il faut vivre "dedans » en prison pour ressentir à quel point la liberté peut être fondamentale. Libres, nous ne prenons jamais la réelle dimension de ce mot. De tous nos aller-retour en prison, nous ne sommes jamais sortis de prison sans ressentir un intense et profond bonheur d'être à l'air libre, « dehors». Souvent, juste au moment de la sortie, nous avons pensé à la chance que nous avons d'appartenir au monde que nous habitons, besoin de nous rassurer peut-être? de nous légitimer ou de cacher la honte? Parfois, juste au moment de la sortie, nous avons réussi à trouver notre vie belle, et à d'autres instants, nous associerons à la prison sa fantastique capacité de nous faire relativiser les problèmes du quotidien qui, "avant la prison ", nous paraissaient insurmontables. Qu'avons-nous fait pour culpabiliser autant? Besoin d'humilité ? C'est parfois l'humour, souvent la dérision, parfois les deux qui s'imposent jusqu'à ce que la raison revienne et que les images, les bruits, les odeurs, les visages s'estompent.

\section{Qu'en est-il de notre légitimité ?}

17 Une autre réflexion tout aussi importante que fortuite voire improbable vient perturber le caractère légitime de notre recherche. «Tant que tu n'as pas été enfermé, et que tu as pas connu toi-même la prison, tu peux pas capter ce que c'est » (Verneris, 
2018, p. 42) nous confie Sylvie qui a passé quatre années en détention, lors du premier entretien. Par cette pensée, elle cherchait probablement à nous alerter, à juste titre, des difficultés que nous allions rencontrer. Cette observation mérite toute sa place; elle nous permet de nous interroger sur notre capacité à comprendre ce que nous n'avons pas vécu pour ensuite analyser et interpréter ce que nous n'avons pas subi. Nous avons consacré, pour cette recherche, presque dix années d'immersion dans le milieu pénitentiaire. Les premières années en tant que formatrice salariée du Greta en région Centre, j'entrais en prison deux à trois journées par semaine pour y rencontrer les détenus qui souhaitaient participer aux dispositifs de formation que l'institution leur proposait. Je travaillais avec ces personnes sur leurs probables modalités de réinsertion dans des conditions d'enfermement identiques aux leurs. Par la suite, ayant changé d'employeur, je me suis tournée vers le bénévolat en proposant des interventions au titre d'ateliers d'écriture, une fois par semaine, une demie journée, profitant des RTT ${ }^{4}$, week-end et périodes de vacances. Pour autant il est vrai que, chaque fois que nous y entrions, nous savions que, quoiqu'il s'y passe, nous sortirions quelques heures après, retrouver notre confortable quotidien. La démarche de biographisation contribue à cet effort de compréhension en nous apportant les éléments empiriques nécessaires à rendre compte du sens que donne chacun à sa vie, à ses actions, à travers son discours. La réponse à nos doutes se trouve probablement dans la qualité de nos observations et explorations in situ, et notre disposition bienveillante à déplacer notre champ de vision pour embrasser les perspectives d'autres personnes que nous-mêmes.

\section{Qu'en est-il de notre déontologie?}

Au risque de surexposer et d'accentuer les situations de précarité dites sensibles sans pouvoir les atténuer, notre démarche bien que compréhensive fait revivre, dans l'épaisseur du vécu biographique, ce que les personnes souhaiteraient peut-être oublier et laisser derrière elles. L'entretien peut être ressenti comme une épreuve "non seulement dans le sens où la personne qui s'y engage accepte l'entretien, mais aussi parce qu'elle se met en demeure de trouver un sens à ce qu'elle fait » (Negroni, 2013, p.61). Et au-delà de la parole échangée, la personne se racontant à elle-même, nous savons que le regard sur soi peut être source de douleur parce qu'elle évoque avec nous des expériences socialement condamnées. Ainsi Fathy nous confie :

Quand je suis devant quelqu'un qui est vrai et qui fait sa vie correctement, (il faut entendre honnêtement) même si j'ai beaucoup plus d'argent que lui, même si je peux l'impressionner avec tout mon argent, peu importe, dans mon propre fond, je sais qu'il est meilleur que moi : (Verneris, 2018, p. 63).

19 Fathy ne se reconnait plus comme appartenant à son monde d'avant, celui de la délinquance, le partage de ses expériences lui a permis d'objectiver sa situation et de trouver sa voie après avoir emprunté d'autres chemins éprouvés.

Mais se raconter c'est aussi faire un détour par le passé, parfois évoquer des souvenirs, et peut se révéler un moment douloureux, certains récits ont été marqués par des moments de tristesse et de larmes. Ainsi Liana, emprisonnée à tort à l'étranger durant quatre ans, essaie de nous parler du viol qu'elle a subi en prison «Ce qui suit est très difficile encore pour moi à raconter, cinq années ont passé depuis, je n'ai rien oublié, mais j'ai toujours autant de mal à en parler ", (p. 171). Ne pas pouvoir mettre de mot sur un vécu traumatique accentue l'angoisse; il nous est arrivé de proposer 
d'interrompre l'entretien, le temps de laisser les émotions s'exprimer. Quand Claude, 80 ans au moment de l'entretien, fait allusion aux violences et sévices infligés par les adultes qui l'encadraient en orphelinat durant son enfance :

Il y a comme un très fort contentieux entre le religieux et moi, je pense qu'il ne s'effacera jamais, les préjudices ont été trop lourds à porter, les blessures n'ont pas cicatrisé, elles sont toujours là, profondes. Le contentieux s'édulcorera probablement avec le temps. Pour l'instant, Il suffit de très peu pour que les choses remontent encore en surface et les nuits sont longues et difficiles. Ce passé, à mon avis, ne s'effacera pas. Ce qui a changé en moi, c'est que je le supporte maintenant, alors que je ne le supportais pas avant: (p. 231).

21 Nous entendons la souffrance, nous la comprenons et nous la respectons. Ainsi nous constatons que, loin d'avoir vécu des évènements similaires à ceux racontés par Fathy, Liana ou Claude, la relation de réciprocité installée entre la personne participante à la recherche et le chercheur, est établie de sorte que chacune des personnes en présence fait « ce qu'il faut » pour accueillir l'autre dans ou depuis son rapport au sensible.

Il n'y a donc ni règle de conduite ni recette prédéterminée à la prise en compte du sensible, les principes méthodologiques et protocoles esquissés dans les manuels de sociologie (Becker, 2004; Quivy, 2006; Payet, 2010; Ogien, 2012; Mucchielli, 2014) conseillent une posture d'empathie respectueuse, favorisant l'écoute attentive, la bienveillance, la neutralité et le non-jugement. De même qu'il n'y a pas de définition simple de "la bonne posture » ou de "la posture la plus adéquate " parce que celle-ci ne peut se caractériser qu'à travers une pluralité de dimensions humaines. Christophe Niewiadomski (2012) évoque une posture qu'il qualifie de «clinique psychosociale»parce que la souffrance que nous côtoyons sur le terrain du sensible interroge: des situations de malaise psychique dont les racines se trouvent dans des situations sociales réelles qui influencent en retour le psychisme des individus, sans qu'il soit pour autant nécessaire d'en référer à la psychopathologie ( $\mathrm{p}$. 129).

23 Il définit la «clinique psychosociale » comme une clinique de l'écoute, de la prise en compte du discours de l'individu et du porter attention à celui-ci, sans passer par la psychologisation des problèmes, " avec les préoccupations déontologiques et éthiques visant à reconnaître chaque individu dans sa pleine dimension d'acteur au sein de son propre développement » (p. 156). Tenter de décrire la manière dont nous avons vécu l'expérience du sensible lors de notre recherche, une manière tout aussi singulière qu'elle nous renvoie à notre histoire, à la manière dont nous communiquons avec le monde, nous donne l'occasion de comprendre ce qui s'y joue. Nous avons le sentiment que, pour laisser toute la place à la parole de l'autre et à sa subjectivité, il nous faut penser avant tout, à installer la confiance dans la relation à l'autre, en nous appuyant sur les valeurs qui fondent notre ouverture perceptive au monde, notre capacité à la compréhension pour, temporairement nous effacer par l'oubli de soi, par une mise entre parenthèse de nos convictions et croyances. Pour autant, la qualité et la crédibilité des résultats imposent au chercheur une posture rigoureuse et impartiale car l'univers carcéral dans lequel nous avons évolué nous a dévoilé les nombreuses polémiques sociétales qu'il suscite, nous y avons découvert des tensions, des passions et révoltes dans lesquelles il est facile de tomber. Nous y avons trouvé des lieux de conflits au milieu d'espaces dangereux et violents qui nous ont amenés à renégocier certaines de nos habitudes de travail, revisiter nos zones de confort quitte à revenir sur les approches et démarches mises à mal évoluant en terrain sensible. 
24 Au-delà de ses ajustements méthodologiques rendus nécessaires par la nature du terrain, la richesse de la méthode se situe dans la justesse de la relation interpersonnelle et la finesse de son articulation. L'éthique de la délicatesse (JannerRaimondi, 2020) requiert ainsi une approche fondamentale pluridimensionnelle, spécifique au champ du sensible impliquant réciprocité, authenticité et sensibilité ; une posture hybride que nous avons favorisée tout au long de cette recherche située en milieu carcéral qui nous a appris à nous surpasser et nous a enrichi. Une posture de chercheur qui associe aux compétences techniques, purement méthodologiques partagées par la communauté des chercheurs en sciences sociales, une dimension humaine s'autorisant à ne pas respecter toutes les méthodes et consignes à la lettre, laissant une part de choix à l'intuition et à l'improvisation.

S'il nous est arrivé de passer parfois du terrain du sensible aux situations de l'extrême, d'autres questionnements ont alors été convoqués comme se demander à partir de quand ou à partir de quoi, l'évènement du sensible peut être considéré comme un évènement traumatique, ou s'interroger sur le regard que porterait la recherche biographique sur la notion d'évènement traumatique? Deux questions qui feront l'objet d'un second article dans ce même ouvrage.

\section{BIBLIOGRAPHIE}

Becker, H. (2004). Ecrire les sciences sociales. Commencer et terminer son article, sa thèse ou son livre. Paris : Economica.

Bois, D. et Austry, D. (2007). Vers l'émergence du paradigme du Sensible. In Réciprocités. Revue du Centre d'Étude et de Recherche Appliquée en Psychopédagogie Perceptive, l'Université Moderne de Lisbonne $1,1-17$.

Boumaza, M. et Campana, A. (2007). Enquêter en milieu « difficile ». Revue française de science politique, 07(1), 5 à 25 repéré à : https://www.cairn.info/revue-francaise-de-sciencepolitique-2007-1-page-5.htm

Delory-Momberger, C. (2009). La condition biographique. Essai sur le récit de soi dans la modernité avancée. Paris : Téraèdre.

Delory-Momberger, C. (2014). De la recherche biographique en éducation. Fondements, méthodes, pratiques. Paris : Téraèdre.

Demazière, D. (2008). L'entretien biographique comme interaction négociations, contreinterprétations, ajustements de sens. Langage et société, 123(1), 15-35. Repéré à : https://doi.org/ $10.3917 / \mathrm{ls} .123 .0015$

Garfinkel, H. (1967). Recherches en ethnométhodologie. Paris : Puf.

Janner-Raimondi, M. (2017). Visages de l'empathie en éducation. Grenoble : Champ Social.

Janner-Raimondi, M. (2020). Processus de biographisation et éthique de la délicatesse dans Expériences vécues et surgissement d'événements : une écriture du sensible en recherche biographique, Questions Vives, recherches en éducation. Argumentaire $\mathrm{N}^{\circ} 34$. 
Laub, D. (2015). Une clinique de l'extrême-le coq-héron 220. Paris : Eres

Mucchielli, L. (2014). Sociologie de la délinquance. Paris : Armand Colin.

Negroni, C. (2013). La latence, concept clé des bifurcations professionnelles : approcher la prise de décision à travers une analyse de récit et du statut de la parole des individus. in Christophe Niewiadomski \& Christine Delory Momberger (dir) Territoires contemporains de la recherche biographique, Teraedre.

Niewiadomski, C. (2012). Recherche biographique et clinique narrative. Entendre et écouter le Sujet contemporain. Toulouse : Eres

Ogien, A. (2012). Sociologie de la déviance. Paris : Puf.

Payet, J.P., Rostaing, C. et Giuliani, F. (2010). La relation d'enquête. La sociologie au défi des acteurs faibles. Rennes : Pur.

Quivy, R. (2006). Manuel de recherche en sciences sociales. Malakof : Dunod.

Ricœur, P. (1991). Lectures 1, autour du politique. Paris : le Seuil.

Rogers, C. (1972). Carl Rogers, Liberté pour apprendre (1e éd. Rév. ; traduit par D. Le Bon). Paris :

Bordas.

Rogers, C. (2001). Carl Rogers, L'approche centrée sur la Personne. Anthologie de textes présentés par Howard Kirschenbaum et Valérie Land Henderson. (traduit pa H.-G. Richon). Lausanne : Randin.

Rogers, C. (2015). Carl Rogers, La relation d'aide et la psychothérapie (19e éd. Rév. ; traduit par J-P Zigliara). Paris : Esf.

Rostaing, C. (2010). De la reconnaissance en prison? Vulnérabilité des ordres de reconnaissance et du mépris. La reconnaissance à l'épreuve. Explorations socio-anthropologiques. Presses Universitaires du Septentrion. 141-148.

Verneris, M.H. (2018). Récits de vie en milieu carcéral, des identités blessées, des vies cabossées. Paris : L'Harmattan.

\section{NOTES}

1. d'autant que certaines d'entre elles se trouvent, au moment des entretiens, encore engagées dans le processus carcéral et judiciaire pour les faits qui leur sont reprochés.

2. Après la seconde guerre mondiale, un nouveau courant de pensée apparait, le modèle positiviste, cherchant à comprendre les causes de la criminalité pour aider à réhabiliter et resocialiser le délinquant. Ce courant de pensée s'oriente vers une description du criminel comme un individu différent du non-délinquant, aux traits de personnalité impulsif, trop « troublé » pour être vraiment rationnel, présentant des prédispositions et un penchant au crime $\mathrm{du}$ fait de certaines déficiences. Les comportements déviants sont rapidement associés à une pathologie affectant certains individus plutôt que d'autres. Pour Cesare Lombroso (2010), professeur de médecine légale italien, le délinquant, la prostituée, le clochard, l'alcoolique, le fou ne peuvent être que " des erreurs de la nature, incapables d'accéder à une vie normale, du fait de leurs caractéristiques biologiques, physiques et parfois héréditaires ". La théorie du déterminisme (biologique, génétique ou neurologique) et tous les préjugés associés vont dominer la compréhension de la déviance jusqu'au milieu du XX ${ }^{\text {ème }}$ siècle. Ce paradigme est à l'origine de nombreuses politiques d'exclusion, de déportation ou d'élimination. 
3. Thèse de doctorat en Sciences humaines et sociales, spécialité Sciences de l'éducation «Démarches de biographisation en milieu carcéral : Quels patrons biographiques en perspective ?»

4. réduction du temps de travail

\section{RÉSUMÉS}

Cet article s'inscrit dans le cadre de la recherche biographique dont le récit est le cœur de sa réflexion théorique. L'auteure intervenant régulièrement en milieu carcéral, auprès de personnes en situation de grande vulnérabilité, s'appuyant sur son expérience, propose de questionner les différentes postures du chercheur confronté à des situations relevant du terrain du sensible, mais aussi de penser la place et le rôle qu'il occupe dans les expériences vécues retransmises. Le terrain du sensible inviterait-il le chercheur à reconsidérer son sens moral et sa crédibilité, à questionner sa responsabilité, sa légitimité, son objectivité et enfin à évaluer sa capacité à voir la souffrance sans pouvoir la réduire, sans prendre la défense et sans prendre parti pour les plus faibles ou pour l'institution qui les enferme? Des questions qui s'appuient sur une démarche de recherche qualitative et qui abordent l'éthique et la déontologie dans une relation humaine, une relation de réciprocité installée de sorte que chacune des personnes en présence lors de l'entretien de recherche fait «ce qu'il faut » pour accueillir l'autre dans ou depuis son rapport au sensible.

This article is part of the biographical research whose narrative life story is the heart of his theoretical reflection. The author regularly intervenes in prison, with people in situations of great vulnerability, proposes to question the different postures of the researcher confronted with situations belonging to the sensitive area, but also to think about the place and the role it occupies in the lived experiences transmitted. Would the sensitive's area invite the researcher to reconsider his moral sense and credibility, to question its responsibility, its legitimacy, its objectivity and finally to evaluate its capacity to see suffering without being able to reduce it, without taking defense and without taking sides for the weakest or for the institution that locks them up? Questions that are based on a qualitative research approach and that address ethical conduct and ethics in a human relationship, an established reciprocal relationship, established so that each person involved in the research interview does "what it takes" to welcome the other into or from his or her relationship to the sensitive.

\section{INDEX}

Keywords : sensitive area, life story, ethical conduct, aréa of ethics, best practice

Mots-clés : terrain sensible, récit de vie, éthique, déontologie, bonne pratique 


\section{AUTEUR}

\section{MARIE HĖLÈNE VERNERIS}

Ingénieure en formation pour adultes, docteure en sciences de l'éducation à l'université Paris Sorbonne Nord, membre du laboratoire scientifique Experice. 